\title{
SOME BLUE STARS OF PECULIAR TYPE IN THE REGION OF THE SOUTH GALACTIC POLE
}

\author{
J. A. G R A H A M \\ 'Cerro Tololo Inter-American Observatory*, Chile \\ and \\ A. SLETTEBAK \\ Perkins Observatory, The Ohio State and Ohio Wesleyan Universities, U.S.A.
}

\begin{abstract}
. $u v b y$ photometric observations have been used in conjunction with slit spectra to classify 90 stars which were noted as peculiar by Slettebak and Brundage in a recent objective prism survey of the South Galactic Pole region. In this paper, we review the photometric classification criteria and identify in the Slettebak-Brundage list, 8 subdwarf $O$ stars, 10 subdwarf B stars, 10 horizontal branch stars, 1 white dwarf star and 26 late subdwarf stars. Three stars with outstanding peculiarities are SB (Slettebak-Brundage) 58 which is a helium subdwarf O star, SB 319 (CD-38 245$)$, a late type star with extremely weak metal lines and SB 845 (BD - 13 $\left.{ }^{\circ} 6465\right)$, an A type star with a very small Balmer discontinuity.
\end{abstract}

\section{Introduction}

Intermediate band photometry can be used very effectively as a means of classifying stars of many different types on the basis of color measurements alone. The increased spectral resolution over broad band photometry allows quantitative measures to be made of such features as the color gradient, the Balmer discontinuity and of some lines and bands in stellar spectra. These photometric indices can be used in turn as classification parameters. In this paper, we report on an application of such a procedure to a group of 90 peculiar high latitude blue stars. These stars were recently found during the course of an objective prism survey for early type stars which was carried out by Slettebak in collaboration with R. K. Brundage (Slettebak and Brundage, 1971). We have obtained in addition slit spectra for the purpose of checking and further refining some of the photometric classifications.

In the Slettebak-Brundage investigation, a $4 \frac{1}{2}^{\circ}$ ultraviolet transmitting prism was used with the Curtis Schmidt telescope at Cerro Tololo Inter-American Observatory to obtain spectra at a dispersion of $580 \AA \mathrm{mm}^{-1}$ (at $\mathrm{H} \gamma$ ) over an area of $840 \mathrm{sq} \mathrm{deg}$ centered on the South Galactic Pole. The limiting magnitude of the plates is between 14 and 15 for the purpose of spectral classification. During the course of the survey, a number of stars with unusual spectral characteristics were noted. These were specifically, (i) all $O$ and $B$ type stars since at the faint limit of the survey, these stars are likely to be subluminous with respect to the main sequence of early type stars, (ii) stars with unusually broad, weak or strong spectral lines for their spectral type. Some of the stars included by the second criterion had spectra later than type F0 which was

* Operated by the Association of Universities for Research in Astronomy, Inc., under contract with the National Science Foundation. 
the limit for listing normal stars, however, these objects were retained because of their special interest as possible faint subdwarf stars.

\section{Photometry and Photometric classification Procedure}

To date, Graham has obtained photometry for 90 stars which were noted as peculiar in the Slettebak-Brundage survey. The intermediate band $u v b y$ system described by Strömgren (1963) and by Crawford and Barnes (1970) has been used for this purpose. Elsewhere, Graham (1970) has outlined a classification scheme which is based on the properties of the $u v b y$ system. This scheme in turn derives from the detailed study of a number of high latitude blue stars by Sargent and Searle (1968). Since the Graham (1970) paper was written, a number of small modifications have been made to the classification criteria. Table I shows the scheme as presently formulated. Note the addition of a separate group to include the late type subdwarf stars. We emphasise here, however, that a photometric classification scheme does have limitations. For example, no distinction can be made between a B type main sequence star and a B type horizontal branch star which has lower mass and lower luminosity but rather similar surface gravity. The differences seen on slit spectrograms such as the variation in helium line intensities have not as yet been detected photometrically. Complications also appear in the classification procedure when heavy interstellar reddening is present. At high galactic latitudes, however, these effects are not serious. Generally speaking, we find that the spectral types estimated from the $u v b y$ photometry are more accurate than those derived from objective prism plates but that the most precise classifications of all follow from the inspection of slit spectrograms especially when complementary photometric data are also available.

\section{TABLE I}

Criteria for $u v b y$ photometric classification

\begin{tabular}{|c|c|}
\hline Type & Classification criteria \\
\hline sd O subdwarf O & $\begin{array}{l}(b-y) \text { very blue, }<-0.120, m_{1}<+0.100 \text { showing Balmer lines are weak } \\
\text { if present. } c_{1}<0.000 \text {. }\end{array}$ \\
\hline sd B subdwarf B & $\begin{array}{l}(b-y)<-0.100,0.100<m_{1}<+0.150 \text { indicating strong Balmer lines } \\
\text { for color, } c_{1}<0.000 .\end{array}$ \\
\hline B & $\begin{array}{l}\text { Colors of unreddened main sequence B star. Category includes stars } \\
\text { with weak helium lines. }\end{array}$ \\
\hline Aw Weak line A & $(b-y)$ and $c_{1}$ of a main sequence $\mathrm{A}$ star, $m_{1}<+0.125$ \\
\hline $\begin{array}{l}\text { AFhb horizontal } \\
\text { branch }\end{array}$ & $\begin{array}{l}c_{1}>1.200 \text { or at least } 0.200 \text { greater than } c_{1} \text { given by standard Hyades } \\
\text { relation (Crawford and Perry, 1966). This is indicative of the low surface } \\
\text { gravity. }(b-y)>0.000 \text {. RR Lyrae stars are included in this group. }\end{array}$ \\
\hline $\begin{array}{l}\text { DA hydrogen line } \\
\text { white dwarf } \\
\text { sd FG late subdwarf }\end{array}$ & $\begin{array}{l}m_{1}>0.150 \text { indicative of very strong hydrogen lines. } c_{1}<0.000,(b-y) \\
<+0.200 \text {. } \\
(b-y)>+0.180, c_{1} \text { normal but } m_{1} \text { less than standard Hyades value by } \\
\text { at least } 0.045 \text {. }\end{array}$ \\
\hline
\end{tabular}




\section{Slit Spectroscopy}

Slit spectrograms have been obtained for 41 of the stars of peculiar type in the Slettebak-Brundage list. These were taken with the Cassegrain spectrograph at the 60 -in. telescope of the Cerro Tololo Inter-American Observatory. 14 of the brightest stars were observed at a dispersion of $39 \AA \mathrm{mm}^{-1}$. These are the stars numbered in the Slettebak-Brundage list (SB) 11, 80, 94, 134, 158, 253, 290, 294, 483, 527, 707, 735, 815,939 . The spectrograms were examined in detail by Slettebak. Notes were made about the differences between these and those of standard stars taken under similar observing conditions. Measurements of radial velocity were also made of each of the $39 \AA \mathrm{mm}^{-1}$ spectrograms. 27 fainter stars were examined also with spectrograms of dispersion $200 \AA \mathrm{mm}^{-1}$. These included several stars whose colors had been particularly difficult to interpret. Again, Slettebak made a detailed comparison between these stars and a number of standard stars and his notes were used in conjunction with the uvby data to classify the stars into the groups listed in Table I. These low dispersion spectrograms were not suitable for radial velocity measurement but a special note was made if the spectrum seemed to be red or blue shifted by a significant amount with respect to the iron arc comparison lines.

\section{The Peculiar Stars in the Slettebak-Brundage List of Early Type Stars}

With the combination of the objective prism, photometric, and slit spectroscopic data, we have classified in Table II the 90 stars for which we have observations. A number of these stars have colors of main sequence $A$ and $F$ stars and these are included as a separate group.

8 subdwarf $\mathrm{O}$ stars are listed. These include one very unusual object, SB 58. Our spectrogram of this star is shown in Figure 1. Strong lines of helium, both neutral and ionised are seen. The Balmer lines are weak and barely visible. A possible large blue shift was noted on the $200 \AA \mathrm{mm}^{-1}$ spectrogram.

10 stars are considered as probable subdwarf B stars. A study of the data from all three sources indicates that the helium lines are almost invariably weak for the color. A typical example of this class, SB 459 (CD - 33 $\left.{ }^{\circ} 417\right)$ is shown in Figure 1. The characteristic broadening of the hydrogen lines with respect to those found in main sequence stars is clearly seen in this spectrogram when a comparison is made with the standard B2 star $\gamma$ Peg. Two stars, SB 7 and SB 744, are unusual in that they have very red $(b-y)$ indices for their class. This suggests that either the stars are composite (however there was no sign of the necessary red companions on our spectrograms) or that the effect is caused by a circumstellar dust shell. Reddening by interstellar material is unlikely because in no single case did any of the more numerous A and F stars in our list show any such color anomaly. In our spectrogram of SB 7 there are traces of a weak K-line but we cannot be sure that this is not a plate flaw.

The group of $14 \mathrm{~B}$ stars contains a mixture of types. Some of the brighter stars appear indeed to be normal main sequence stars (SB 80, SB 158). SB 253 and SB 463 

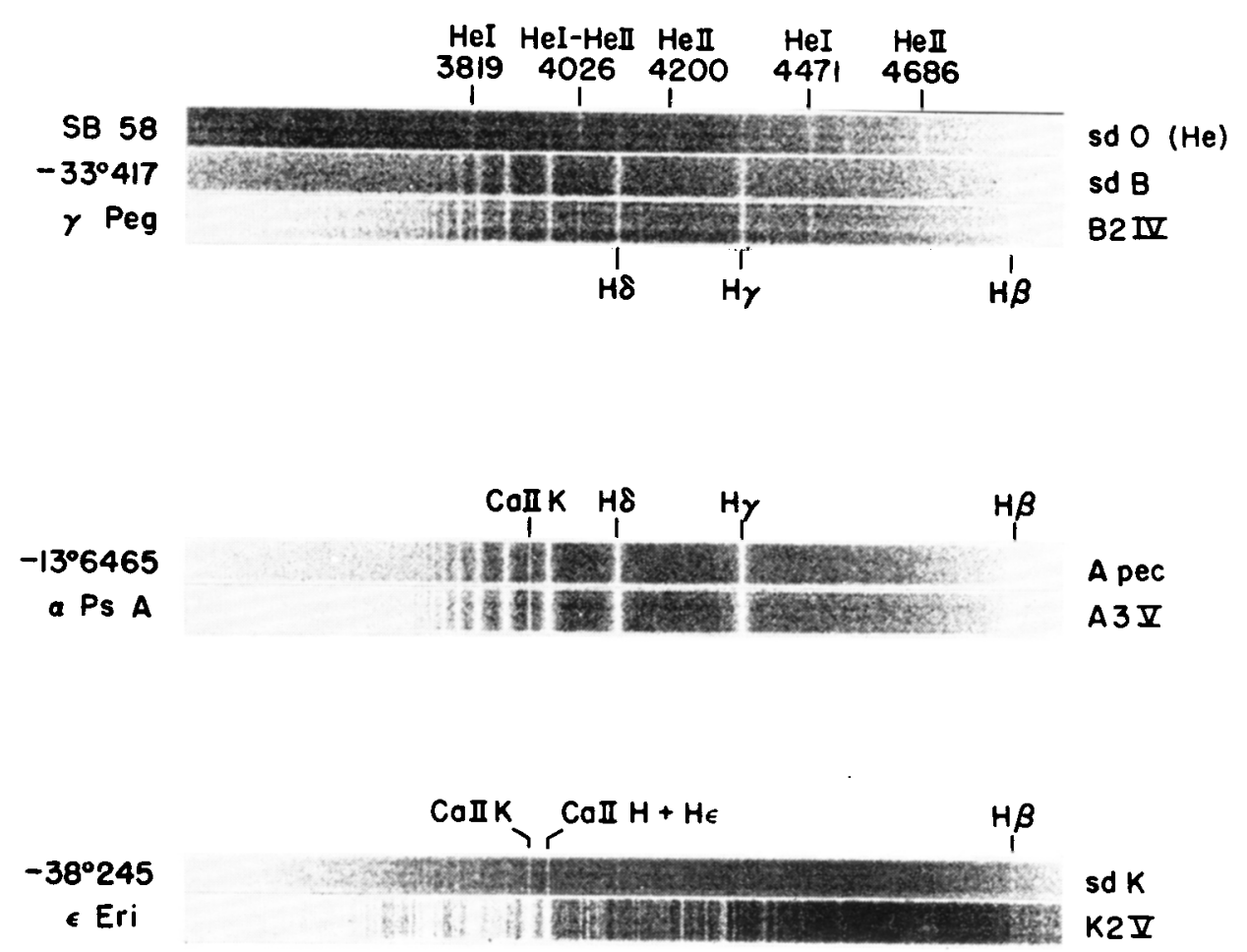

Fig. 1. Spectrograms of peculiar high latitude stars compared with spectrograms of bright standard stars.

have normal spectra but probable high velocities and are most likely runaway stars from the galactic plane. SB 395 and SB 460 appear to be B stars with weak helium lines and similar to those classified as Bw by Sargent and Searle (1968). SB 527 with high velocity, narrow lines and spectral type B8 is a probable horizontal branch star with lower surface gravity than a main sequence star of similar type.

One at first surprising result of our work is the very small number of horizontal branch $\mathrm{A}$ and $\mathrm{F}$ stars that are found. Of the 10 stars classified as horizontal branch AF stars, 8 are probable RR Lyrae stars. 5 of these are variables which were discovered prior to our study and it is interesting to note that all 8 probable RR Lyrae stars are identifiable as horizontal branch stars on the basis of color measurements alone and without knowledge of their variability. When average colors are plotted on a $(b-y)-c_{1}$ diagram, it is found that the RR Lyrae variables fall on the continuation of the sequence occupied by non-variable horizontal branch stars. The almost complete absence of the A-type horizontal branch stars in our list of peculiar types can be explained by the fact that these stars do not show conspicuous spectroscopic anomalies at the dispersion used in the objective prism survey. This was deduced after examining in detail a sample of 17 stars classed as (normal) early A type. These stars 
TABLE II

Classification of 90 blue stars of peculiar type in the Slettebak-Brundage list

Type

sdO

$\operatorname{sdB}$

B

AFhb

sdFG

AF

Peculiar Slettebak-Brundage number

$\begin{array}{lrrrrrrrrrr}38 & 58^{\mathrm{a}, \mathrm{f}} & 147 & 169 & 705 & 884 & 931^{\mathrm{b}} & 933 & & & 8 \\ 7^{\mathrm{c}, \mathrm{d}} & 8^{\mathrm{e}} & 290^{\mathrm{c}} & 360 & 410^{\mathrm{c}} & 459^{\mathrm{c}} & 485^{\mathrm{c}} & 707^{\mathrm{c}} & 744^{\mathrm{cd}} & 815^{\mathrm{c}} & 10 \\ 21 & 80^{\mathrm{e}} & 158^{\mathrm{e}} & 171 & 253^{\mathrm{e}, \mathrm{f}} & 357^{\mathrm{f}, \mathrm{g}} & 395^{\mathrm{c}, \mathrm{f}} & 446 & 460^{\mathrm{c}, \mathrm{f}} & 14 \\ 463^{\mathrm{e}, \mathrm{f}} & 527^{\mathrm{p}, \mathrm{h}} & 735 & 774 & 939 & & & & & \end{array}$

$\begin{array}{lllllllllll}31^{1} & 107 & 338^{\mathrm{i}} & 405^{\mathrm{i}} & 406^{\mathrm{i}} & 453^{\mathrm{i}} & 714^{\mathrm{i}} & 830 & 900^{\mathrm{i}} & 909^{\mathrm{i}} & 10\end{array}$

$\begin{array}{lllllllllllll}26 & 44 & 68 & 178 & 264 & 273 & 274 & 319 & 330 & 342 & 421 & 26\end{array}$

$\begin{array}{lllllllllll}442^{\mathrm{P}} & 469 & 501 & 606 & 623 & 683 & 730 & 919 & 941 & 961^{\mathrm{P}} & 962\end{array}$

$963 \quad 965 \quad 966 \quad 967$

702

$\begin{array}{llllllllllll}11^{\mathrm{j}} & 51 & 94^{\mathrm{k}} & 115 & 134^{\mathrm{l}} & 189 & 260 & 282^{\mathrm{l}} & 294^{\mathrm{m}} & 349 & 419 & 20\end{array}$

$\begin{array}{llllllllll}483^{1} & 495 & 540 & 553^{\mathrm{P}} & 712 & 718 & 719 & 720 & 803\end{array}$

845

No. of stars

I SB 58. Slit spectrogram shows very strong $\mathrm{He}$ I and $\mathrm{He}$ II but very weak Balmer lines. Helium subdwarf O star. Colors red for a sd O star.

b SB 931. $(b-y)$ red for a sd O star. No spectrogram available.

c Probably weak helium lines.

d $(b-y)$ unusually red for classification which is based on slit spectrogram.

e No unusual features in spectrum. Normal spectral type.

P Possible high velocity. Slight red or blue shift noted on $200 \AA \mathrm{mm}^{-1}$ spectrum.

g SB 357. Balmer lines rather weak. $\mathrm{H} \beta$ not visible suggesting presence of line emission.

h SB 527. Slit spectrum indicates B8 but Balmer lines are sharp. Horizontal branch star?

i RR Lyrae variable.

j SB 11. Peculiar A-star of the silicon type.

k SB 94. Strong $\mathrm{Sr}$ II, similar to late Am or $\beta \mathrm{CrB}$ star.

1 Metallic line A-star.

m SB 294. $\lambda$ Bootis star, weak metal lines, low velocity, moderate rotation.

have apparent magnitudes close to 13. uvby observations have been made of these stars and it is found that of the 17, 7 show the definite characteristics of horizontal branch stars. In the Slettebak-Brundage list, there are 159 early A stars of 13 th mag. The small sample observed suggests that between 50 and 80 of these are horizontal branch stars. It is clear, therefore, that if $u v b y$ observations had been made of the entire Slettebak-Brundage list, many more horizontal branch A stars would have been detected.

26 late type subdwarf stars seem to be present in our list. Perhaps the most interesting of these is SB 319 (CD - 38 245$)$ which may be a very cool, extreme member of the class. The $200 \AA \mathrm{mm}^{-1}$ spectrogram that we have is reproduced in Figure 1. Only the $\mathrm{H}$ and $\mathrm{K}$ lines are prominent. The Balmer lines are very sharp and weak and no other lines are visible. The uvby photometry $\left(y: 12.00,(b-y):+0.580, m_{1}:+0.070\right.$, $\left.c_{1}:+0.409\right)$ is suggestive of a very metal deficient $\mathrm{K}$ star. The comparison with the spectrogram of the K2 standard star in Figure 1 indicates how extreme this deficiency may be. A second very peculiar star in our list is SB 845 (B.D. - 13 ${ }^{\circ} 6465$ ). We are unable to classify this star into any of the categories in Table I. It appears as a peculiar 
A star with weak Balmer lines at objective prism dispersion. The $u v b y$ photometry is itself peculiar $\left(y: 10.74,(b-y):+0.106, m_{1}:+0.123, c_{1}:+0.352\right)$. The $(b-y)$ and $m_{1}$ indices are normal for an early $\mathrm{A}$ star but the Balmer discontinuity is very small. Our slit spectrogram, reproduced in Figure 1, shows a weak but definite K-line (equivalent to type A2) and weak Balmer lines with no other lines visible. The very small Balmer discontinuity can be seen in Figure 1 from the comparison with the A3V star, $\alpha$ PsA. It does not seem likely that the star is composite, since no combination of two stars seems capable of producing the observed colors and spectrum. There is no sign of Balmer emission either line or continuum. The star is not faint and we feel that it would merit a very much more detailed investigation.

\section{References}

Crawford, D. L. and Perry, C. L.: 1966, Astron. J. 71, 206.

Crawford, D. L. and Barnes, J. V.: 1970, Astron. J. 75, 978.

Graham, J. A.: 1970, Publ. Astron. Soc. Pacific 82, 1305.

Sargent, W. L. W. and Searle, L.: 1968, Astrophys. J. 152, 443.

Slettebak, A. and Brundage, R. K.: 1971, Astron. J. 76, 338.

Strömgren, B. 1963, in K. Aa. Strand (ed.), Basic Astronomical Data, University of Chicago Press, Chicago, p. 123. 